See discussions, stats, and author profiles for this publication at: https://www.researchgate.net/publication/325853980

\title{
Evaluation of inflammatory processes by FTIR spectroscopy
}

Article in Journal of Medical Engineering \& Technology · June 2018

CITATIONS

0

6 authors, including:

Lais Carvalho

São Paulo State University

8 PUBLICATIONS 10 CITATIONS

SEE PROFILE

2. Franck Bonnier

University of Tours (François-Rabelais) - UFR pharmaceutical sciences

72 PUBLICATIONS 1,496 CITATIONS

SEE PROFILE

Some of the authors of this publication are also working on these related projects:

COSMICC View project

Renata Bitar View project
READS

156

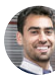

Luis Felipe Das Chagas e Silva de Carvalho

Universidade de Taubaté

40 PUBLICATIONS 141 CITATIONS

SEE PROFILE

Ana Lia Anbinder

São Paulo State University

95 PUBLICATIONS 597 CITATIONS

SEE PROFILE 


\section{Evaluation of inflammatory processes by FTIR spectroscopy}

\section{Laís Morandini Rodrigues, Luís Felipe das Chagas e Silva Carvalho, Franck Bonnier, Ana Lia Anbinder, Herculano da Silva Martinho \& Janete Dias Almeida}

To cite this article: Laís Morandini Rodrigues, Luís Felipe das Chagas e Silva Carvalho, Franck Bonnier, Ana Lia Anbinder, Herculano da Silva Martinho \& Janete Dias Almeida (2018): Evaluation of inflammatory processes by FTIR spectroscopy, Journal of Medical Engineering \& Technology, DOI: $10.1080 / 03091902.2018 .1470691$

To link to this article: https://doi.org/10.1080/03091902.2018.1470691

曲 Published online: 04 Jun 2018.

Submit your article to this journal $₫$

Q View related articles $\longleftarrow$

View Crossmark data \lceil 


\title{
Evaluation of inflammatory processes by FTIR spectroscopy
}

\author{
Laís Morandini Rodrigues ${ }^{a}$, Luís Felipe das Chagas e Silva Carvalho ${ }^{\mathrm{b}, \mathrm{c}}$, Franck Bonnier ${ }^{\mathrm{d}}$, Ana Lia Anbinder ${ }^{\mathrm{a}}$, \\ Herculano da Silva Martinho ${ }^{e}$ and Janete Dias Almeida ${ }^{a}$ \\ aapartment of Bioscience and Oral Diagnosis, Institute of Science and Technology of São José dos Campos, Univ Estadual \\ Paulista-UNESP, São José dos Campos, Brazil; 'b Departamento de Odontologia, Universidade de Taubaté, Taubate, Brazil; \\ 'Graduação em Odontologia, Universidade Braz Cubas, Mogi das Cruzes, Brazil; ${ }^{\mathrm{d}}$ Faculty of Pharmacy, Université François-Rabelais \\ de Tours, Tours, France; ${ }^{e}$ Centro de Ciências Naturais e Humanas, Universidade Federal do ABC, Santo Andre, Brazil
}

ABSTRACT

Fourier transform infrared (FTIR) spectroscopy is a powerful diagnosis technique and has been used to identify patterns of molecular changes based on vibration modes. The objective of this study was to evaluate inflammatory fibrous hyperplasia (IFH) lesions and oral normal mucosa (NM) initially with histopathological exam and then using micro-FTIR spectroscopy to analyse the samples. Eleven IFH and 11 NM samples were analysed at five different points to cover the largest area possible by the micro-FTIR technique. Bands were observed between 970 and $1743 \mathrm{~cm}^{-1}$ which corresponded to different structural components like collagen, lipids, fatty acids, proteins and amino acids. Spectral bands were more intense mostly for IFH lesions, including collagen bands, which are an important component of inflammatory fibrous hyperplasia. This study demonstrated that differentiation in the inflammatory tissue was observed in FTIR spectral differences, in terms of biochemical composition.

\section{ARTICLE HISTORY}

Received 29 December 2017

Revised 10 April 2018

Accepted 18 April 2018

Published online 31 May

2018

\section{KEYWORDS}

FTIR spectroscopy;

inflammatory proc-

esses; diagnosis

\section{Introduction}

Advances in diagnostic techniques including Fouriertransform infrared (FTIR) spectroscopy have permitted the rapid and efficient analysis of pathological tissues and has received great attention from the medical community for the high accuracy in monitoring the structural changes in normal and pathological samples (biological fluids, cells and tissues) [1-3].

This technique should therefore become a routine diagnostic tool as it allows a real time and non-invasive diagnosis. And, since time is a determinant factor for the best prognosis of a disease, FTIR spectroscopy can provide a clear, objective and rapid diagnosis for many different diseases.

FTIR spectroscopy is based on the absorption of infra-red light by vibrational transitions of covalent bonds which provides information about all macromolecules [4], as DNA, RNA, proteins and carbohydrates present in the cells $[5,6]$. This means the technique can give biochemical information directly linked to biological properties. A benefit of this method is that samples do not require any staining or preparation and can be analysed as soon as removed; avoiding loss of relevant biochemical information [7].
Tian et al. [8] proved that FTIR spectroscopy was an efficient method for breast cancer diagnosis. The accuracy of the technique was $94 \%$ and the authors concluded that it can be considered an auxiliary technique for histopathological diagnosis. Teophilou et al. [9] also used the technique for the differentiation of benign versus malignant ovarian lesions. They classified the FTIR spectroscopy technique as an efficient method to detect malignant tissues as well as to determine the type of carcinoma.

Inflammatory fibrous hyperplasia (IFH) is one of the most common injuries in clinical routines [10]. Its occurrence is linked to chronic trauma of low intensity in patients with complete dentures [11] and is a lesion with no potential for malignant transformation. However, it does require surgical removal, histopathological diagnostic confirmation and correct clinical management.

Currently, histopathology is considered the gold standard for diagnosis [12]. However, according to Tian et al. [8] histopathology has many limitations, since time is required for fixation, cutting, staining and analysis by a pathologist. For these reasons, the patient has to wait a considerable time for a final

CONTACT Laís Morandini Rodrigues lais.morandini@gmail.com Instituto de Ciência e Tecnologia de São José dos Campos - UNESP, Departamento de Biociências e Diagnóstico Bucal, Av. Francisco José Longo, 777 São Dimas, 12245-000, São José dos Campos, São Paulo, Brazil

(C) 2018 Informa UK Limited, trading as Taylor \& Francis Group 


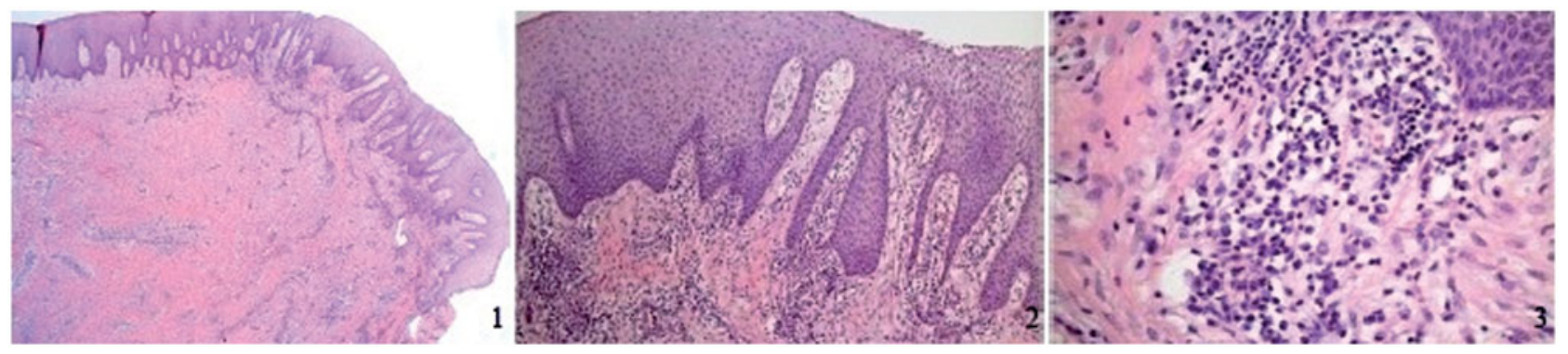

Figures 1-3. (1) Parakeratinised stratified pavement epithelium with hyperplasia of epithelial cones and dense fibrous connective tissue. (2) Presence of the moderate chronic inflammatory infiltrate. (3) Hydropic degeneration, acanthosis and inflammatory cells like plasmocytes and lymphocytes.

diagnosis. Also, the diagnosis is subjective and based upon the experience and knowledge of the pathologist [8].

In medical science there are numerous lesions that require additional procedures in order to be properly diagnosed. Many of the methods used to diagnose these lesions are not sufficient to reach a conclusive diagnosis. New techniques are necessary to supplement the final diagnosis and help clinicians find the correct treatment for the benefit of the patient.

In dentistry, few studies involving FTIR spectroscopy have been done due to unawareness of the new technique. In this way, the aim of this study was to evaluate the effectiveness of FTIR spectroscopy as a diagnostic tool for the diagnosis of oral mucosal IFH lesions compared to normal oral mucosa.

\section{Materials and methods}

The study was approved by the Ethics Committee of the Institute of Science and Technology, UNESP - Univ Estadual Paulista, São José dos Campos, São Paulo, Brazil, Protocol number 032/2010-PH/CEP. The research was conducted in accordance with the guidelines of the National Health Council. The patients received detailed information about the study and agreed to the collection of tissue samples.

\subsection{Sample preparation}

Biopsy specimens were collected from oral mucosa of 11 patients with a differential diagnosis of IFH at the Department of Biosciences and Oral Diagnosis, Institute of Science and Technology, Univ Estadual Paulista - UNESP, São José dos Campos, Brazil. Samples from normal mucosa (NM) were obtained from the same patients with IFH during surgery, with extension of the incision in at least $3 \mathrm{~mm}$ to ensure an injury-free area. All biopsies were excised with scalpel in order to preserve intact the delimitation between NM and IFH. Part of the lesion was sent to histopathological examination to confirm the final diagnosis of IFH and the presence of injury-free area (NM).

The IFH samples that were analysed by FT-IR spectroscopy had $0.5 \mathrm{~cm}$ in length and thickness of $2 \mathrm{~mm}$ approximately, while NM had $2 \mathrm{~mm}$ length and same thickness. The samples were washed in $0.9 \% \mathrm{NaCl}$ $\left(\right.$ Aster $\left.^{\circledR}\right)$, transferred to properly identified and numbered cryogenic vials (Nalgene ${ }^{\circledR}$ ) and immediately stored in a freezer (Thermo Scientific Ltd., Waltham, MA) at $-80^{\circ} \mathrm{C}$.

The specimen remained thawing for approximately 3 min in saline solution $\left(0.9 \% \mathrm{NaCl}\right.$, Aster $\left.^{\circledR}\right)$ and then was transferred to a platinum substrate with clinical tweezers. These set were immediately analysed on micro-FTIR spectrometer for the collection of spectral data using an attenuated total reflection (ATR) accessory.

\subsection{Histopathological analysis}

For histopathological analysis, the samples were fixed in $10 \%$ formalin and were stained with haematoxylin and eosin (H\&E) to reveal the tissue structures of the samples.

Histological analysis of NM specimens showed a normal-appearing mucosal lining, lamina propria with collagen fibres arranged in parallel and cellular components characteristic of NM. IFH samples (Figures 1-3) exhibited parakeratinised stratified squamous epithelium, hyperplasia of the basal layer, formation of epithelial cones and dense fibrous connective tissue. In general, all IFH lesions exhibit the same pattern of alterations. The inflammatory infiltrate varied from moderate to intense, as well as the amount of collagen matrix. The samples were analysed by at least two different pathologists. 


\subsection{FTIR spectroscopy}

FTIR spectra were acquired in a Varian $660 / 610$ spectrometer equipped with an germanium attenuated total reflection (ATR) accessory. The samples were placed on a platinum plate and positioned on microscope FTIR. The ATR tip was positioned to touch the samples with a slight pressure to ensure complete contact with germanium crystal. Spectral data were the result of 400 scans, with a spectral resolution of $4 \mathrm{~cm}^{-1}$ covering the spectral window $900-1800 \mathrm{~cm}^{-1}$. A background spectrum was also recorded in air (800 scans) and automatically compared with the sample spectrum by the software. Between each analysis of the samples the crystal was thoroughly cleaned with $70 \%$ ethanol to avoid cross contamination and interferences in the spectra. The spectra were collected in five different locations to ensure the complete analysis of the samples. Ultimately, $55 \mathrm{IFH}$ and $55 \mathrm{NM}$ spectra each were obtained from 22 samples. Considering the different steps involved, the total time for each sample analysis was approximately five minutes.

\subsection{Spectral analysis}

All collected spectra were stored by the equipment control software and converted to ASCII format. FT-IR spectra were converted to csv format and then baseline was corrected by FITyK ${ }^{\circledR}$ software. The different pre-processing and data analysis steps were performed using Matlab (Mathworks Inc., Natick, MA). Processed using baseline correction (rubber-band) followed by vector normalisation (VN).

\subsection{Data analysis}

Principal components analysis (PCA) is a method of multivariate analysis broadly used with datasets of multiple dimensions [13-15]. It allows the reduction of the number of variables in a multidimensional dataset, although it retains most of the variation within the dataset. The order of the principal components (PCs) denotes their importance to the dataset. PC1 describes the highest amount of variation, PC2 the second highest, and so on $[16,17]$.

PCA scatter plot groups similar datasets (spectra) according to the loadings of the PCs and can be used to distinguish different datasets (samples). The loadings represent the variance for each variable (wavenumber) for a given PC. Analyzing the loadings of a PC can give information about the source of the variability inside a dataset, in the case of spectroscopy, derived from variations in the molecular components contributing to the spectra. PCA was employed in this study to differentiate the spectroscopic signatures of the two samples analysed, IFH and NM.

Linear discriminant analysis (LDA) used for classification and discrimination purposes between groups was performed using Minitab software. The LDA technique is a supervised analysis, as the classes already have a pre-defined identity. A sample classified in a group is the closer observation of the Mahalanobis distance to the centre of the group, characterised by average. For each group there is a distance which is known as linear discriminant function. Thus, samples giving a smaller distance for the linear discriminant function will be classified as belonging to it. To avoid errors in the classification of samples in each group, the leaveone-out cross-validation technique was applied at the LDA.

This technique is used to generate a new classification function, where the Mahalanobis distance is recalculated for each sample. In this case, a sample is always omitted and is calculated as a new classification function to see if the data are still the same groups previously classified. Thus, all samples will have their recalculated Mahalanobis distance, reducing error rates.

\section{Results}

The mean spectra and standard deviation of the groups could be observed in Figure 4(A). Bands correlated with vibrational modes and structural components are shown in Table 1, assigned to each peak observed. The frequencies of the bands shows qualitative features about the nature of the chemical bands, their structures and molecular environment. Bands corresponding to polysaccharides, nucleic acids, collagen, amides, lipids, protein, amino acids and fatty acids were observed.

After spectra normalisation, mean and standard deviation were calculated and box plots were constructed for NM and IFH samples. Figure 4(B) shows discrimination between samples and correlation between bands that present greater variations by simple analysis of the box plots (Red line - NM, Black Line - IFH). The spectral bands are numbered (Table 1) in order to establish the analysis of differences between the two groups. It is possible to notice that there are differences in the intensity of bands related to IFH samples, which mostly showed higher intensity than the bands of NM tissue. However in some instances the peaks present the same intensity and sometimes the intensity was higher in NM samples, as in the peaks 1743,1643 and $1544 \mathrm{~cm}^{-1}$ which corresponds 

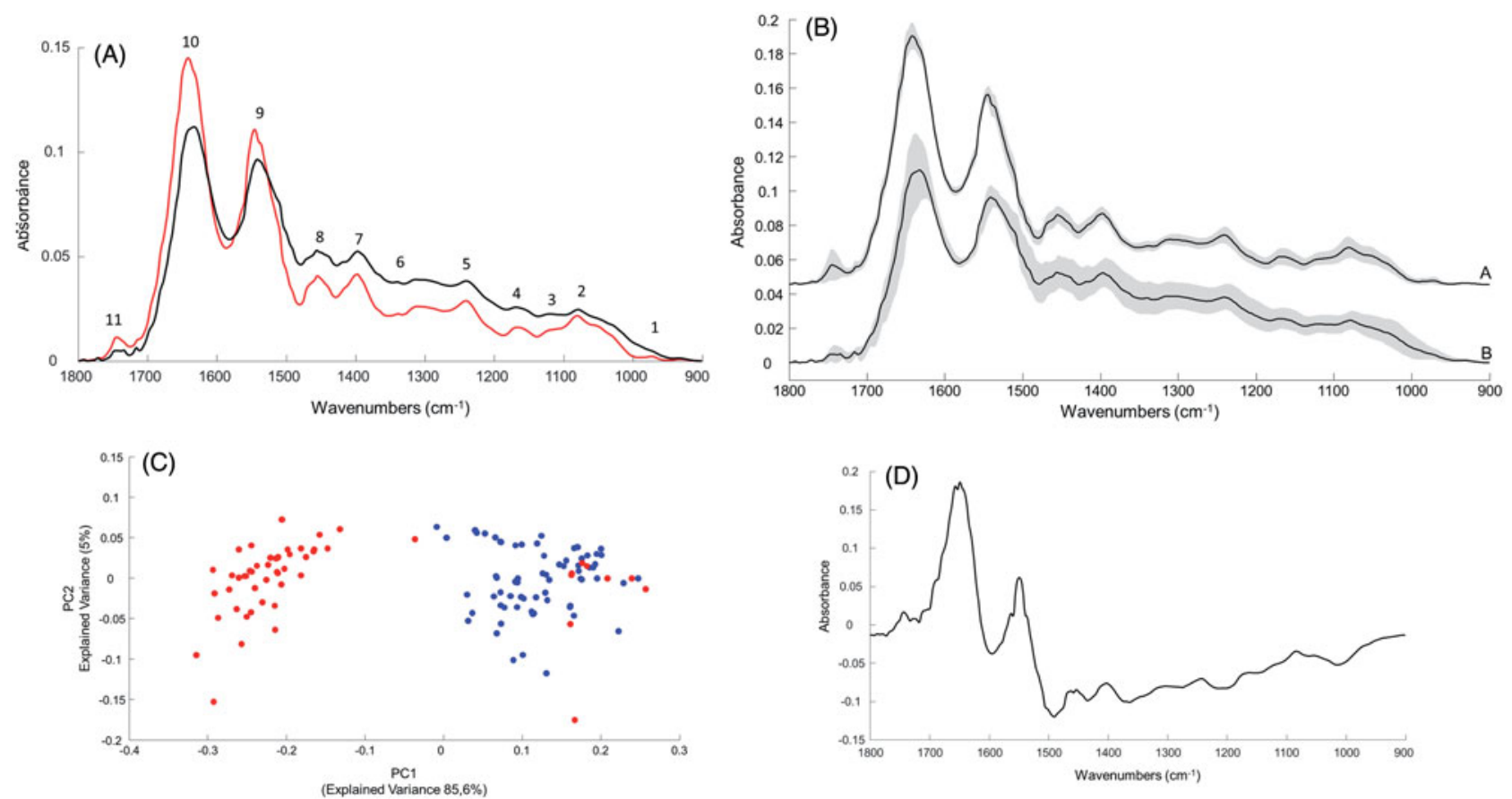

Figure 4. (A) Mean spectra and the standard deviation of the groups. (B) Discrimination between samples and correlation between bands that present greater variations by simple analysis of the box plots: Red line - NM, Black Line - IFH. (C) PCA analysis show well defined discrimination between the two groups, NM spectra are in blue and IFH in red. (D) The loading corresponding to the PC 1.

Table 1. Correlation between bands, vibrational modes, structural components and ANOVA. ${ }^{\text {a }}$

\begin{tabular}{|c|c|c|c|}
\hline Band $\left(\mathrm{cm}^{-1}\right)$ & Vibrational mode & Structural component & ANOVA $(p<.05)$ \\
\hline 1. 970 & $\begin{array}{l}\text { Symmetric stretching mode of dia- } \\
\text { nionic phosphate monoesters }\end{array}$ & $\begin{array}{l}\text { Phosphorylated proteins or cellular } \\
\text { nucleic acids - DNA. }\end{array}$ & $<.05$ \\
\hline 2. 1080 & $\begin{array}{l}\text { Phosphate vibration } \\
\text { Symmetric } \\
\text { phosphate }\left[\mathrm{PO}_{2}^{-}(\text {sym)] stretching }\right.\end{array}$ & $\begin{array}{l}\text { Collagen and phosphodiester groups } \\
\text { of nucleic acids. }\end{array}$ & $<.05$ \\
\hline 3. $1163 / 4$ & C-O stretching band & $\begin{array}{l}\text { Glycogen } \\
\text { Collagen }\end{array}$ & $<.05$ \\
\hline 4. 1172 & $\begin{array}{l}\text { Stretching vibrations of nonhydrogen- } \\
\text { bonding } \mathrm{C}-\mathrm{OH} \text { groups } \\
\mathrm{CO} \text { stretching }\end{array}$ & Collagen & $<.05$ \\
\hline 5. 1240 & $\mathrm{~V}_{a s} \mathrm{PO}_{2}^{-}$ & Collagen & $<.05$ \\
\hline 6. 1312 & Amida III & Protein & $<.05$ \\
\hline 7. 1398 & $\mathrm{CH}_{3}$ symmetrical deformation & - & $<.05$ \\
\hline 8. 1454 & Asymmetric methyl deformation & - & $<.05$ \\
\hline 9. 1553 & $\begin{array}{l}\mathrm{CO} \text { stretching } \\
\text { Amide II band mainly stems from } \\
\text { the } \mathrm{C}-\mathrm{N} \text { stretching and } \mathrm{C}-\mathrm{N}-\mathrm{H} \\
\text { bending vibrations weakly coupled } \\
\text { to the } \mathrm{C}=\mathrm{O} \text { stretching mode. }\end{array}$ & - & $<.05$ \\
\hline 10. 1643 & $\begin{array}{l}\text { Amide I band (arises from } \mathrm{C}=0 \\
\text { stretching vibrations) }\end{array}$ & - & $<.05$ \\
\hline 11. 1743 & $\mathrm{C}=\mathrm{O}$ stretching mode & Lipids & $<.05$ \\
\hline
\end{tabular}

${ }^{\mathrm{a} D i e m}$ et al. [24]; Movasaghi et al. [25]

to lipid, amide I and II bands. All these results indicate the presence of alterations in different molecules that are associated with the characteristics of the IFH process, including collagen, a typical feature of IFH injuries.

The coupling of FTIR spectroscopy with multivariate analysis such as PCA is a powerful combination to highlight and interpret the variation in the data sets collected. For instance, when comparing the MN spectra to the IFH spectra based on an unsurprised method such as PCA, it is interesting to whiteness the normal well defined discrimination between the two groups (Figure $4(C)$ ). NM spectra are in blue and IFH in red. While the PC 1 represents $85.5 \%$, PC 2 only 
Table 2. Confusion matrix of PCA-LDA results.

\begin{tabular}{llrr}
\hline & \multicolumn{2}{c}{ Histology } \\
\cline { 2 - 4 } & IFH & & NM \\
\hline Spectroscopy & 70 & & 0 \\
IFH & 10 & & 45 \\
NM & & 87.5 & \\
Sensitivity (\%) & & 100 & \\
Specificity (\%) & & \\
\hline
\end{tabular}

accounts for $5 \%$ and it is obviously not contributing in grouping the spectra. The loading corresponding to the PC 1 (Figure 4(D)) give an overview of the different wavenumbers involved in the separation observed between the two groups with main features at 970, 1080 and $1172 \mathrm{~cm}^{-1}$, respectively assigned to nucleic acids, glycogen and collagen. Although, the discrimination between the two groups is well pronounced, a few of the IFH spectra remains not recognised as pathological and is grouped with the NM spectra. This can be explained by the presence of heterogeneity in the biopsies with some that can present different pathological process of inflammation or due to the experimental set up. The ATR measurement remains the delicate operation and the correlation of the measurements with precise location on the biopsies is difficult. Therefore, it should be considered that few of the repetition have been performed close to the lesion border where the tissue is normal. It is one of the main reasons to have performed recording on five different locations to cover as much as possible of the biopsies surface. Ultimately, in order to get classification rates in terms of sensitivity and specificity, a supersized alternative, PCA-LDA, has been employed. Using a cross validation sequence leaving a part of the data out at each interaction, the classification of the spectra can be calculated and a confusion matrix was constructed, as displayed in Table 2. The sensitivity was $87.5 \%$ and specificity was $100 \%$, demonstrating the approach deliver good accuracy for the detection of IFH tissues.

\section{Discussion}

The study demonstrated the diagnostic capability of FTIR spectroscopy to effectively differentiate between inflammatory and NM samples, indicating the nature of the molecular changes. Analysis of spectral band intensity showed important differences between groups in all bands analysed, particularly with regard to collagen important feature found on IFH lesion, when the tissue is analysed by H\&E.

Differently from Fernandez et al. [18], who constructed tissue microarrays from archival specimens with prostate cancer samples and applied FTIR microspectroscopic imaging to provide both structural detail and spectral information indicative of tissue biochemistry, in our study fresh tissues (ex vivo) were analysed to evaluate effectiveness of the technique (FTIR spectroscopy) without H\&E staining, for a future application in vivo and in real time.

IFH is characterised by cell proliferation in an organ or tissue, including the oral cavity and may be caused by low-intensity chronic mechanical irritation $[19,20]$. In the oral cavity, the lesion is characterised histopathologically by an increase in the number of inflammatory cells [21] and the connective tissue becomes more fibrous mainly due to an increase in collagen production, with maintenance of the morphofunctional pattern of the tissue.

In this respect, we believe that the increase in the fibroblastic cells, which are responsible for collagen production, the increase in their capacity of replication and the presence of inflammatory cells (lymphocytes) leads to an increase in the amount of DNA molecules, which is evidenced by an increase in intensity of bands at 970 and $1080 \mathrm{~cm}^{-1}$ in IFH samples.

Although the inflammatory component (lymphocytes) of IFH is not the most prominent component of the lesion and its amount may vary, it is important to consider that lymphocytes may interfere in the interpretation of normal or malignant spectra, presenting false-positive or false-negative results [22]. The interpretation of the data of inflammatory component allows the creation of a spectral database that may eventually contribute to the in vivo diagnosis.

The significance of infra-red spectral signature of human lymphocytes is extensively documented in the literature, notably delivering useful information to assess the effectiveness of immunotherapies [4] and possible correlation with survival rates [23] in patients diagnosed with carcinoma.

The band at $1080 \mathrm{~cm}^{-1}$ can also be attributed to glycogen [24,25]. It is known that this molecule is linked to the production of energy. When needed, glycogen is broken down through the process of glycolysis, generating pyruvate, which is then metabolised to form acetyl-CoA. This molecule fuels the citric acid cycle with the accompanying production of ATP molecules [26]. In their study, Weisberger and Fischer [27] showed that alteration of glycogen metabolism and phosphorylation of related proteins can be the underlying causes of keratinisation in oral mucosa, while Goltz et al. [28] attributed the increase of glycogen to increased cell differentiation in carcinoma. This is an important property for the classification of the disease. In our case, we believe that an increase in the quantity of this molecule can be associated with 
the increase of the cellular proliferation, which are fibroblastic cells and the increase of the production and secretion of collagen, which requires an extra charge of energy.

Another marked feature of IFH is the presence of significant amounts of collagen fibre bundles in connective tissue [20] as a result of an increase in fibroblast numbers, since these cells produce the extracellular matrix, that means an excessive deposition of collagen and increase in the clinical volume of these lesions [29]. Observing the data obtained in this study, histopathological findings are confirmed by deposition of collagen fibres replacing the connective tissue. Collagen was observed in bands at 1081 and $1240 \mathrm{~cm}^{-1}$ in which its intensity is higher for the IFH. An opposite pattern was found by the authors Bodanese et al. [30] who observed decreased collagen bands in basal cell carcinoma in the spectroscopic characterisation that was attributed to its degradation.

The bands at 1500 and $1600 \mathrm{~cm}^{-1}$ correspond to amide I and II and are related to the presence of protein (peptides). The amide I band is mainly associated with $\mathrm{C}=\mathrm{O}$ stretching of peptide groups and the amide II bands are associated with $\mathrm{C}-\mathrm{N}$ stretching and $\mathrm{N}-\mathrm{H}$ angular deformation, both from peptide groups too [31,32]. The same is observed for amide III bands, with deformation of the CO-NH group. The band in $1743 \mathrm{~cm}^{-1}$ is related to the lipid. In fact, the composition of plasmatic cell membrane bilayers is compound of phospholipids with associated proteins. The increase in intensity of the bands above mentioned indicates the maintenance of cell integrity in NM, which is different from hyperplastic epithelium in which cells have morphologic changes and deformations.

The aim of the study was specifically to demonstrate that ATR-FTIR has enough sensitivity to detect molecular variations in inflammatory process. There is a consensus among the authors who studied the spectroscopy techniques and there are no limitations in its application. Some studies with biofluids like saliva and blood have shown the presence of water-related bands that may interfere with the results, but this limitation has been solved by drying the samples before analysis, as observed in the studies of Rodrigues et al.; Bottoni et al.; Khaustova et al. and Petibois et al. [33-36].

The main benefit for spectroscopy technique application: is in vivo and real-time tissue analysis without surgical removal. In terms of the effectiveness, FTIR spectroscopy can be considered much more accurate than the histopathological examination, which although is considered the gold standard diagnosis, is subjective because depends on the evaluation of the pathologist who may have different opinions about the same pathological process. Also, complementary tests are sometimes required, e.g. immunohistochemistry, to confirm the diagnosis. This all takes time and in some cases, time is crucial for good prognosis. Furthermore, surgical procedure in some individuals may cause stress and sometimes it is necessary to use medications (tranquilisers) in order to perform the procedure. For all these reasons is extremely interesting to study optic biopsy techniques for in vivo and real-time application.

Ultimately the identification of specific spectral markers in IFH lesions suggest that the technique is able to detect the delimitation of the lesion to guide the surgeons and ensure that adequate margins have been applied during the excision of the pathological area. This is a crucial observation especially for lesion with high recurrence as malignant lesions which require precise evaluation of the security margins to ensure the entire lesion has been removed and consequently improve prognostic for the patients.

FTIR spectroscopy was able to discriminate normal tissue from IFH based on the superficial molecular information collected from the ATR tip. Considering the recent developments made in IR fibre optics and ATR probe, the present study demonstrated the feasibility to transfer the methods to in vivo guidance of the surgeons during tissue resection [37]. We highlight the diagnostic potential of FTIR spectroscopy based on the effectiveness of the technique to characterise the vibrational properties in the IFH samples studied.

\section{Conclusion}

FTIR spectroscopy is efficient in providing molecular data of inflammatory processes, permitting the quantitative and qualitative analysis of the molecular composition of normal and pathological tissues by revealing the vibrational modes of functional groups that are important for the differentiation between them. The technique allowed analysis of molecular changes observed in inflamed tissue and effective characterisation, showing that the technique can be an important tool in clinics.

\section{Acknowledgments}

The authors would like to acknowledge the patients who took part in this study, and Multiusers Central Facilities of UFABC for the experimental support.

\section{Disclosure statement}

No potential conflict of interest was reported by the authors. 


\section{Funding}

This work was supported by grants 2010/15363-3 and 2011/ 0668-0, São Paulo Research Foundation (FAPESP), Coordination for the Improvement of Higher Education Personnel (CAPES), and Brazilian National Council for Scientific and Technological Development (CNPq).

\section{References}

[1] Giorgini E, Tosi G, Conti C, et al. FTIR microspectroscopic characterization of Spitz nevi. Spectrochim Acta A Mol Biomol Spectrosc. 2015;141:99-103.

[2] Dukor RK, Vibrational spectroscopy in the detection of cancer. Chichester: John Wiley \& Sons Ltd; 2002.

[3] Eikje NS, Aizawa K, Ozaki Y. Vibrational spectroscopy for molecular characterisation and diagnosis of benign, premalignant and malignant skin tumours. Biotechnol Annu Rev. 2005;11:191-225.

[4] Wald N, Legat A, Meyer C, et al. An infrared spectral signature of human lymphocyte subpopulations from peripheral blood. Analyst. 2015;140:2257-2265.

[5] Diem M, Romeo M, Boydston-White S, et al. A decade of vibrational micro-spectroscopy of human cells and tissue (1994-2004). Analyst. 2004;129:880-885.

[6] Pevsner A, Diem M. Infrared spectroscopic studies of major cellular components. Part II: the effect of hydration on the spectra of nucleic acids. Appl Spectrosc. 2001;55:1502-1505.

[7] Hollywood KA, Maatje M, Shadi IT, et al. Phenotypic profiling of keloid scars using FT-IR microspectroscopy reveals a unique spectral signature. Arch Dermatol Res. 2010;302:705-715.

[8] Tian P, Zhang W, Zhao H, et al. Intraoperative diagnosis of benign and malignant breast tissues by Fourier transform infrared spectroscopy and support vector machine classification. Int J Clin Exp Med. 2015;8:972-981.

[9] Theophilou G, Lima KM, Martin-Hirsch PL, et al. ATRFTIR spectroscopy coupled with chemometric analysis discriminates normal, borderline and malignant ovarian tissue: classifying subtypes of human cancer. Analyst. 2016;2:585-594.

[10] Santos PP, Nonaka CF, Pinto LP, et al. Immunohistochemical expression of mast cell tryptase in giant cell fibroma and inflammatory fibrous hyperplasiaof the oral mucosa. Arch Oral Biol. 2011;3: 231-237.

[11] Kiuchi M, Yamamura T, Okudera $M$, et al. An assessment of mast cells and myofibroblasts in dentureinduced fibrous hyperplasia. J Oral Pathol Med. 2014; 1:53-60.

[12] Conti C, Ferraris P, Giorgini E. Microimaging FT-IR of oral cavity tumours. Part III: cells, inoculated tissues and human tissues. J Mol Struct. 2006;834: 86-94.

[13] Varmuza K, Introduction to multivariate statistical analysis in chemometrics. 2nd ed. New York: Taylor \& Francis Group; 2009.

[14] Korenius T, Laurikkala J, Juhola M. Information sciences on principal component analysis, cosine and
Euclidean measures in information retrieval. Information Sciences. 2007;177:4893-4905.

[15] German MJ, Hammiche A, Ragavan N, et al. Infrared spectroscopy with multivariate analysis potentially facilitates the segregation of different types of prostate cell. Biophys J. 2006;10:3783-3795.

[16] Kelly JG, Trevisan J, Scott AD, et al. Biospectroscopy to metabolically profile biomolecular structure: a multistage approach linking computational analysis with biomarkers. J Proteome Res. 2011;4:1437-1448.

[17] Martin FL, German MJ, Wit E, et al. Identifying variables responsible for clustering in discriminant analysis of data from infrared microspectroscopy of a biological sample. J Comput Biol. 2007;9:1176-1184.

[18] Fernandez DC, Bhargava R, Hewitt SM, et al. Infrared spectroscopic imaging for histopathologic recognition. Nat Biotechnol. 2005;23:469-474.

[19] Canger EM, Celenk P, Kayipmaz S. Denture-related hyperplasia: a clinical study of a Turkish population group. Braz Dent J. 2009;20:243-248.

[20] Firoozmand ML, Almeida JD, Cabral LAG. Study of denture-induced fibrous hyperplasia cases diagnosed from 1979 to 2001. Quintessence Int. 2005;10: 825-829.

[21] Coelho CM, Zucoloto S, Lopes RA. Denture-induced fibrous inflammatory hyperplasia: a retrospective study in a school of dentistry. Int J Prosthodont. 2000;2:148-151.

[22] Carvalho LF, Bitar RA, Arisawa EA, et al. Spectral region optimization for Raman-based optical biopsy of inflammatory lesions. Photomed Laser Surg. 2010; 28:S111-S117.

[23] Wald N, Bordry N, Foukas PG, et al. Identification of melanoma cells and lymphocyte subpopulations in lymph node metastases by FTIR imaging histopathology. Biochim Biophys Acta. 2016;1862:202-212.

[24] Diem M, Griffiths P, Chalmers J, Vibrational spectroscopy for medical diagnosis. 1st ed. Chichester (UK): John Wiley; 2008.

[25] Movasaghi Z, Rehman S, Rehman IU. Raman spectroscopy of biological tissues. Appl Spectrosc Rev. 2008;43:134-179.

[26] Gazi E, Dwyer J, Gardner P, et al. Applications of Fourier transform infrared microspectroscopy in studies of benign prostate and prostate cancer. A pilot study. J Pathol. 2008;201:99-108.

[27] Weisberger D, Fischer CJ. Glycogen content of human normal buccal mucosa and buccal leukoplakia. Ann $\mathrm{N}$ Y Acad Sci. 1960;85:349-350.

[28] Goltz RW, Fusaro RM, Jarvis J. Observations on glycogen in epithelial tumors. J Invest Dermatol. 1958;31:331-341.

[29] de Oliveira HC, Tschoeke A, da Cruz GC, et al. MMP-1 and MMP-8 expression in giant cell fibroma and inflammatory fibrous hyperplasia. Pathol Res Pract. 2016;212:1108-1112.

[30] Bodanese B, Silveira FL, Zângaro RA, et al. Discrimination of basal cell carcinoma and melanoma from normal skin biopsies in vitro through Raman spectroscopy and principal component analysis. Photomed Laser Surg. 2012;30:381-387. 
[31] Vidal Bde C, Mello ML. Collagen type I amide I band infrared spectroscopy. Micron. 2011;42:283-289.

[32] Belbachir K, Noreen R, Gouspillou G, et al. Collagen types analysis and differentiation by FTIR spectroscopy. Anal Bioanal Chem. 2009;395:829-837.

[33] Rodrigues LM, Magrini TD, Lima CF, et al. Effect of smoking cessation in saliva compounds by FTIR spectroscopy. Spectrochim Acta A Mol Biomol Spectrosc. 2017; 174:124-129.

[34] Bottoni U, Tiriolo R, Pullano SA, et al. Infrared saliva analysis of psoriatic and diabetic patients: similarities in protein components. IEEE Trans Biomed Eng. 2016;2:379-384.
[35] Khaustova S, Shkurnikov M, Tonevitsky E, et al. Noninvasive biochemical monitoring of physiological stress by Fourier transform infrared saliva spectroscopy. Analyst. 2010;12:3183.

[36] Petibois C, Gionnet K, Gonçalves $M$, et al. Analytical performances of FT-IR spectrometry and imaging for concentration measurements within biological fluids, cells, and tissues. Analyst. 2006;131: 640-647.

[37] Li QB, Xu Z, Zhang NW, et al. In vivo and in situ detection of colorectal cancer using Fourier transform infrared spectroscopy. World J Gastroenterol. 2005; 11:327-330. 